\title{
Late Onset Mania in Dengue Fever
}

\author{
Shailendra Mohan Tripathi ${ }^{1, *}$, Neeti Mishra ${ }^{2}$ \\ ${ }^{1}$ Department of Geriatric Mental Health, King George's Medical University, Lucknow, India \\ ${ }^{2} J u n i o r$ Resident, Era's Lucknow Medical College, Lucknow, India \\ *Corresponding Author: dr_smtripathi@rediffmail.com
}

Copyright (C) 2014 Horizon Research Publishing All rights reserved.

\begin{abstract}
Here, we are reporting a case of late onset mania in a 61 year old male secondary to dengue fever. The past and family history of the mood disorder was absent in the patient. Episode of mania had a temporal correlation with the dengue fever. Psychiatric co-morbidities are mentioned in the literature following dengue fever but the literature on the mania following dengue is sparse. The late onset mania in dengue fever is unreported in the literature.
\end{abstract}

Keywords Dengue Fever, Mania, Late Onset

\section{Introduction}

Dengue fever (DF) commonly known as break-bone fever is one of the major health problems in South East Asia including India. The word dengue is derived from African word denga which means fever with hemorrhage. Dengue Fever is a benign syndrome caused by several arthopod borne viruses, which is characterized by high grade fever, Myalgia, or Arthralgia, rash, Leukopenia and lymphadenopathy. In a small proportion of cases the disease develops into the life-threatening dengue hemorrhagic fever(DHF), resulting in bleeding, low levels of blood platelets and blood plasma leakage, or into dengue shock syndrome (DSS), where dangerously low blood pressure occurs.

Dengue fever is the most common arthropod borne viral disease. Dengue fever is one of the most important emerging diseases of the tropical and sub tropical regions, affecting urban and pre urban areas. Recently its root has spread to the rural areas as well. Dengue is emerging as a major public health problem in India. Since the first epidemic in Kolkata during 1963-64 many places in India have been experiencing dengue infection [1].The geographical distribution of the disease has greatly expanded and the number of cases has increased dramatically in the past 30 Years. The increase of Dengue and Dengue Hemorrhagic Fever is due to uncontrolled population growth and urbanization without appropriate water management and inappropriate vector control programme. By 1997 most of the countries have experienced large out breaks of the disease, currently DF / DHF is endemic in Bangladesh, India, Indonesia, Maldives, Srilanka, Thailand approximately 1.3 billion people are living in the endemic areas. Dengue / DHF are widely prevalent in India and all the 4 serotype are found. India has witnessed many epidemic outbursts in the recent past and with each and every episode dengue poses threatening situation to inhabitants of that locality [2]. There has been a decline in dengue incidence after 1996. However during 2001 out breaks have been reported from Rajasthan (1433 cases and 33 deaths), Tamilnadu (761 cases and 8 deaths), Karnataka (161 cases) Gujarat (46 cases). Now a day's most of the northern India faces the problem of dengue outbreaks at the end of each rainy season.

Initially, the dengue was thought to harbor the risk of hemorrhage secondary to fall in the platelet count but nowadays it is also responsible for the encephalitis and encephalopathy $[3,4]$. Late onset mood disorders, including mania, have generally been reported to be associated with vascular risk factors[5]. The mania in older adult secondary to dengue fever is to the best of our knowledge is unreported in the literature. Written informed consent was taken from the patient and his family to present and publish the case for the benefit of the scientific community. Patient's name is written as Mr. A to maintain confidentiality.

\section{Case History}

Mr. A, a 61-year-old male, a resident of Barabanki district of UP, India, developed an episode of mania after an attack of Dengue fever. He consulted the Department of Geriatric Mental Health, King George's Medical University with a history of overactivity, overtalkativeness, argumentativeness, abusiveness, religiosity, sexually preoccupied and a decreased need for sleep of 7days duration. He used to stay up for most of the night. At night he would mumble and sing for long periods. The patient also endorsed hearing voices of his dead wife asking him to join her in heaven. The patient's family was concerned for his safety, and hence his son petitioned him to this institution for hospitalization. Six days prior to the onset of the psychiatric illness, he developed high grade fever $\left(40.1^{\circ} \mathrm{C}\right)$ which was associated with chills and 
rigor, severe headache, confused behaviour, generalized body ache, anorexia and occasional nausea and vomiting. The history of pruritus, photophobia, seizure and concealed or overt bleeding was absent.

On third day of the fever serological test for the NS-1 antigen was positive. The test did not reveal the presence of the IgM or IgG antibodies. His platelet count was 62,000 per microlitre and a TLC- of $5100 / \mathrm{mm} 3$ with $47 \%$ was shared by lymphocytes. The other blood investigations were within normal limits except liver function test. SGOT was 342 $\mathrm{IU} / \mathrm{ml}$ and SGPT was $366 \mathrm{IU} / \mathrm{ml}$, much above than normal range. His blood smear did not revealed malaria parasite. His prothrombin time was 12 seconds, but his bleeding time was slightly increased (6 minutes). He was diagnosed as a case of Dengue fever. The patient was advised to take plenty of the fluid, fruit juices and rest. $\mathrm{He}$ was prescribed only paracetamol as per requirement. He was counseled to get admitted when consecutive blood tests would reveal fall in platelet count. Fortunately his platelet count did not fell significantly. The least platelet count was 52000 and he started showing signs of the recovery on $5^{\text {th }}$ day onwards. On the $6^{\text {th }}$ day of the illness, the patient showed signs of manic symptoms, as discussed above. The family and past history for the mania was absent. There was no history of the hypertension and diabetes mellitus. He had no history of any known chronic illness. On the day of admission, the patient appeared of his stated age, with poor grooming and hygiene, poor eye contact, and he was easily distracted and restless. His speech was spontaneous and effusive; his mood was euphoric; and his affect was wide ranging, dramatic, and labile. The patient's thought content was delusional and was significant for religious and sexual themes simultaneously. The patient had poor insight into his illness and had poor judgment. Physical examination did not reveal any neurological deficit. The patient's BP was 130/80 mm Hg. His pulse rate was 98 per minute and his urine output was 1.2 litre/day. His score on the Young Mania Rating Scale (YMRS) [6] was 26. His Mini Mental State Examination revealed no cognitive impairment, with a score of $28 / 30$. On investigations after admission his blood parameters were within normal limits except liver function test and platelet count. The SGOT was $136 \mathrm{IU} / \mathrm{ml}$ and SGPT was $141 \mathrm{IU} / \mathrm{ml}$. Platelet count was found to be 1.2 lacs per microlitre. DEN specific IgM antibody was detected in the serum of the patient using ELISA kit that confirms the diagnosis of dengue fever. The CT scan of his head was found to be normal. He was successfully treated with sodium valproate $1000 \mathrm{mg}$ per day and quitiapine $300 \mathrm{mg}$ per day.

\section{Discussion}

The patient was diagnosed as a case of mood disorder with manic features, according to DSMIV-TR [7].The temporal correlation of the occurrence of the mania with dengue fever is suggestive that mania was secondary to the dengue fever. The absence of any manic, depressive episode in the past and negative family history in the patient points towards the organic mood disorder. One should also look for indicators like perplexed affect, marked aggression/irritability, cognitive impairments, marked disinhibition, emotional incontinence and other symptoms suggestive of organicity in patients with mania. The patient had confusional state which is very commonly seen in the elderly having fever.

Although bipolar disorder can have a late onset in persons over the age of 50 without a previous psychiatric history or a family history of bipolar disorder.[8,9]. New-onset mania in older adults is most commonly secondary $[10,11]$. As the case mentioned here has first episode mania, so possibility of the secondary mania is always there.

In addition, patients whose first manic episode is after the age of 58 exhibit increased cognitive impairment, which is partially reversible $[12,13]$. Thus, it is important to rule out delirium and dementia.

Mania is typically characterized by an abnormally elevated or irritable mood lasting at least 1 week. It may be accompanied by one or more of the following: grandiosity, decreased need for sleep, increased talkativeness, flight of ideas, distractibility, increased goal-directed activity, psychomotor agitation, and excess involvement in potentially harmful activities, all of which lead to a marked decrease in the level of functioning. These features distinguish themselves from delirium, in which the cardinal feature is a waxing and waning alteration in consciousness accompanied by a change in cognition (e.g., disorientation or memory or language disturbances). The symptoms of delirium may be accompanied by changes in effect, such as anxiety and fear. Differentiation between the two is accomplished by longitudinal observation as well as monitoring for affective changes. This patient had manic symptoms without any waxing or waning of consciousness or other evidence of delirium, which suggests a diagnosis of mania.

The cognitive dysfunction that often accompanies mania in older adults [14] may suggest a diagnosis of dementia. However, there are differences in the presentations and premorbid histories of dementia and mania. Agitation and psychosis in dementia are typically phenomena that occur later in the course of illness, rather than in the initial presentation $[15,16]$.

In a review of 50 consecutive psychiatric admissions for mania of people over the age of 65 , Tohen et al. [17] found that 12 of 14 cases of first-episode mania were related to a neurological disorder or infection, most commonly stroke. Fujikawa et al. [18] also suggested that most cases of secondary mania in older adults result from stroke. However, the incidence of post-stroke mania is low and has been estimated at $1 \%$ of all strokes [19]. There was no evidence of stroke on the CT.

Neurological involvement may occur because of intracranial haemorrhage, cerebral oedema, hyponatremia, cerebral anoxia, fulminant hepatic failure with portosystemic encephalopathy, renal failure or release of toxic products. Pathophysiology of neurological involvement may include 
the following factors: direct tissue lesion caused by the virus because of its neurotropicity, capillary haemorrhage, disseminated intravascular coagulation and metabolic disorders [20]. The exact mechanism by which brain insult leads to mania is unclear, although there is evidence of associations between right-sided lesions and mania[21, 22].

Secondary mania in older adults is a serious medical condition that requires a comprehensive differential diagnosis. Older adults are more susceptible to disorders that can lead to secondary mania, so a thorough past psychiatric history is essential. Late-onset bipolar disorder is possible, but it is not the most likely etiology in older adults [17]. In view of this Mr. A had mania secondary to the dengue fever. The search for the cause behind the mania or any other kind of psychiatric disorder would prevent the patient from receiving prophylactic treatment for the long time.

A better planned study is required to assess the psychiatric co-morbidities in dengue fever as it is one of the very common febrile illnesses in the South-East Asia. At the same time it will be learning curve that if a person comes with first episode mania especially in old age, one should explore the etiology.

\section{REFERENCES}

[1] Bandyopadhyay S, Jain DC, Datta KK: Reported incidence of dengue/DHF in India 1991-1995.DengueBulletin , 20:33-34,1996.

[2] Singh NP, Jhamb R, Agarwal SK, Gaiha M, Dewan R, Daga MK, et al. The 2003 outbreak of Dengue fever in Delhi, India. Southeast Asian J Trop Med Public Health ;36:1174-78, 2005.

[3] Ferreira ML, Cavalcanti CG, Coelho CA, Mesquita SD. Neurological manifestations of dengue: study of 41 cases. ArqNeuropsiquiatr.;63:488-93, 2005. Palma-da Cunha-Matta A, Soares-Moreno SA, Cardoso-de Almeida A, Aquilera-de Freitas V, Carod-Artal FJ. Neurological complications arising from dengue viral infection. Rev Neurol;39:233-37, 2004.

[4] Cassidy F, Carroll BJ. Vascular risk factors in late onset mania. Psychol Med; 32:359-62,2002.

[5] Young RC, Biggs JT, Ziegler VE, Meyer DA. A rating scale for mania: Reliability, validity and sensitivity. $\mathrm{Br} \mathrm{J}$ Psychiatry;133:429-35, 1978.

[6] American Psychiatric Association. Diagnostic and Statistical
Manual of Mental Disorders. 4th. Washington DC: American Psychiatric Association; pp. 345-428. Text Revision (DSM-IV-TR),2000.

[7] Depp CA, Jin H, Mohamed S, Kaskow J, Moore DJ, Jeste DV: Bipolar disorder in middle-aged and elderly adults: is age of onset important? J Nerv Ment Dis ; 192:796-799, 2004.

[8] Depp CA, Jeste DV: Bipolar disorder in older adults: a critical review. Bipolar Disord ; 6:343-367, 2004.

[9] Krauthammer C, Klerman GL: Secondary mania: manic syndromes associated with antecedent physical illness or drugs. Arch Gen Psychiatry ; 35:1333-1339, 1978.

[10] Hoblyn JC, Brooks JO: Herbal supplements in older adults: consider interactions and adverse events that may result from supplement use. Geriatrics ; 60:18, 22-23, 2005.

[11] Young RC, Klerman GL: Mania in late life: focus on age at onset. Am J Psychiatry ; 149:867-876, 1992.

[12] Mirchandani IC, Young RC: Management of mania in the elderly: an update. Ann Clin Psychiatry ; 5:67-77,1993.

[13] Young RC: Geriatric mania. Clin Geriatr Med ; 8:387-399, 1992.

[14] Lopez OL, Becker JT, Sweet RA, Klunk W, Kaufer DI, Saxton J, Habeych M, DeKosky ST: Psychiatric symptoms vary with the severity of dementia in probable Alzheimer's disease. J Neuropsychiatry Clin Neurosci ; $15: 346-353,2003$.

[15] Schneider LS, Dagerman KS: Psychosis of Alzheimer's disease: clinical characteristics and history. J Psychiatr Res ; 38:105-111,2004

[16] Tohen M, Shulman KI, Satlin A: First-episode mania in late life. Am J Psychiatry ; 151:130-132, 1994

[17] Fujikawa T, Yamawaki S, Touhouda Y: Silent cerebral infarctions in patients with late-onset mania. Stroke; 26:946-949, 1995.

[18] Starkstein SE, Boston JD, Robinson RG: Mechanisms of mania after brain injury: 12 case reports and review of the literature. J Nerv Ment Dis; 176:87-100,1998.

[19] Lum LC, Lam SK, Choy YS et al. (1996) Dengue Encephalitis: aTrue Entity? American Journal of Tropical Medicine and Hygiene 54, 256-259.

[20] Mendez MF: Mania in neurologic disorders. Curr Psychiatry Rep; 2:440-445,2000.

[21] Shulman KI: Disinhibition syndromes, secondary mania and bipolar disorder in old age. J Affect Disord ; 46:175-182, 1997. 